\title{
La articulación de teoría, trabajo de campo y formación del antropólogo en Ángel Palerm.Transfiriendo aprendizajes de México a España*
}

\author{
Carlos Giménez Romero
}

Se habla de la influencia en España de las aportaciones de Ángel Palerm en el trabajo de campo en la formación de los antropólogos. El texto toca la génesis del enfoque, el aprendizaje por parte del autor, su aplicación en España y el significado de todo ello. El autor asimiló esa pedagogía como estudiante de antropología en España, como doctorando en la Universidad lberoamericana de México y como investigador en un proyecto orientado según las pautas anteriores. Organizó en la Universidad Autónoma de Madrid el Seminario de Investigación Antropológica, desarrolló proyectos de investigación y prácticas de campo donde participaron licenciados y estudiantes avanzados. Los lineamientos mencionados fueron aplicados y desarrollados para dar lugar a una manera de unir formación e investigación, teoría y trabajo de campo, que hoy perdura.

PALABRAS CLAVE: enseñanza del trabajo de campo, investigación en España, investigación en México, Universidad Iberoamericana, Universidad Autónoma de Madrid

\section{Ángel Palerm's Articulation of Fieldwork, Theory and Training: Transferring Lessons from Mexico to Spain}

This article is about the influence in Spain of Ángel Palerm's contributions regarding the fieldwork as part of anthropological training. I describe how I came to this approach, first in Spain as an undergraduate student of anthropology and later in Mexico as a postgraduate and predoctoral scholar. Back to Spain, as a professor at the Universidad Autónoma de Madrid, I organized the Seminar of Anthropological Research, carrying out research projects and fieldwork practices in which recent graduates and undergraduates participated. The guidelines described above were applied and developed, giving rise to a habit of joining training and research, theory and fieldwork that lasts to the present.

KEYWORDS: field research teaching, research in Spain, research in Mexico, Universidad Iberoamericana, Madrid Autonomus University

Carlos Giménez Romero: Departamento de Antropología, Universidad Autónoma de Madrid, Madrid, España carlos.gimenez@uam.es

Desacatos, núm. 45, mayo-agosto 2014, pp. 47-62

Recepción: 9 de noviembre de 2012 / Aceptación: 30 de septiembre de 2013

\footnotetext{
*Agradezco profundamente a Juan Vicente Palerm y a Laura González la información y comentarios, así como a Marisol Pérez Lizaur el trabajo de edición.
} 


\section{INTRODUCCIÓN}

$\mathrm{L}$ a finalidad de este artículo es hacer un recuento de la influencia en España de las aportaciones de Ángel Palerm (España, 1917-México, 1980) en la realización del trabajo de campo como parte de la formación de los antropólogos, y en la unión de aprendizaje, teoría y estancia de campo. El enfoque palermiano sobre el aprendizaje del trabajo de campo se gestó en México hacia 1970, mediante la labor de un elenco de profesores y alumnos, primero en la Universidad Iberoamericana (UIA) de la ciudad de México y luego en la Universidad Autónoma Metropolitana-Iztapalapa (UAM-I) y en el Centro de Investigaciones y Estudios Superiores en Antropología Social (CIESAS), instituciones en las que Palerm fundó e impulsó proyectos innovadores y de excelencia en antropología. Algunos de los rasgos más sobresalientes de aquel enfoque de inspiración palermiana, compartido y desarrollado por profesores, investigadores y alumnos, aún vigende determinados proyectos de investigación, promovidos por lo general en determinadas regiones y siguiendo la concepción de los estudios de área (Steward et al., 1995); 2) la apertura de estaciones de campo en algunas comunidades de esas regiones, como la estación de la UIA en Tepetlaoxtoc; 3) estancias periódicas en el campo con profesores y alumnos; 4) recorridos y ejercicios colectivos de observación, comentados y supervisados; 5) escritura intensiva y pautada de los diarios de campo por parte de los estudiantes, y comentario oral y escrito por parte de los profesores; 6) elaboración individual y colectiva de fichas temáticas según las pautas de clasificación de la Guía de Murdock (Palerm y Palerm, 1976); 7) supervisión in situ de los diarios y fichas por parte de los profesores y monitores; 8) organización y mantenimiento de un fondo documental colectivo, y 9) discusión de los materiales de campo a partir de las teorías estudiadas. Con esta referencia pretendo situar el relato de la experiencia. Claro que cada universidad, grupo de investigación y época tuvo $-y$ tiene aún - sus rasgos diferenciadores.

\section{ANTECEDENTES: PRIMERA FASE DE APRENDIZAJE EN ESPAÑA (1972-1978)}

Durante mis estudios de antropología en España aprendí una parte notable del enfoque teórico y metodológico de Palerm. Le debo estas enseñanzas teóricas y prácticas al profesor Juan Vicente Palerm, del Departamento de Antropología y Etnología de América de la Universidad Complutense de Madrid (UCM). Juan Vicente, hijo de Ángel Palerm, se trasladó a España en 1964 y era profesor del Departamento cuando comencé a estudiar antropología en el curso 1972-1973. Se dedicaba - y sigue haciéndolo, ahora en la Universidad de California, Santa Barbara (UCSB) - a los estudios campesinos. Cuando lo conocí había realizado trabajo de campo sobre el campesinado en diversas localidades de Jaén, Segovia, Burgos y sobre todo Galicia. Además, era difusor en España de las aportaciones de la antropología mexicana, en concreto de la obra de su padre.

Juan Vicente Palerm nos hacía leer y comentar los textos de Chayanov, Banfield, Redfield, Foster, Wolf y Shanin, entre otros. Por entonces estaba construyendo una "tipología de comunidades rurales". Presentó una primera aproximación a esa tipología en la Primera Reunión de Antropólogos Españoles, celebrada en Sevilla en 1973. Aparte de la obra de referencia de Wolf (1966), Los campesinos, en ese programa de investigación y en esa propuesta de tipología había una nítida perspectiva comparativa, un enfoque regional, ${ }^{1}$ y se utilizaban los "niveles de integración sociocultural” que propusiera Steward en la década de 1950 (Palerm, J. V., 1973: 79-97). Convencido de la conveniencia y la necesidad de prácticas etnográficas y de breves estancias de campo durante la carrera, Palerm organizaba salidas al campo con los estudiantes, en las cuales participé. Esas actividades, junto a otras que luego mencionaré, se llevaron a cabo en el marco del Seminario de

\footnotetext{
${ }^{1}$ Por ejemplo, se utiliza y se cita la obra de Roca-Sastre (1972), $L a$ región y el desarrollo.
} 
Estudios Campesinos que Palerm fundó e impulsó con la colaboración de un grupo de estudiantes. Recuerdo esas salidas al campo, por ejemplo la de Segovia, a localidades sobre el Río Pirón.

Aquellos recorridos me sirvieron para concretar la temática de mi tesina - la propiedad comunal de la tierra- y el área de trabajo de campo. Como ya dije, de esto se trataba el trabajo de campo durante la carrera y la discusión de los textos teóricos, las observaciones y la práctica sobre el terreno. En otro de los recorridos de campo con Palerm llegué al Valle de Valdelaguna, en la región pinariega de las provincias de Burgos y Soria, área en la cual realizaría trabajo de campo durante 1974, 1977 y 1978. Esa visita, y otras, estaban inspiradas en la obra de Costa. El maestro nos motivó no sólo a leer éstos y otros textos de finales del siglo xIX, sino a visitar algunas de las comunidades de las que Costa recibió informe. Posteriormente decidí trasladarme a México y comparar su régimen comunal agrario con el que había estudiado en Valdelaguna y la región pinariega, lo que dio lugar a mi tesis doctoral, un ejercicio de antropología comparada entre México y España (Giménez, 1978, 1991).

Quiero resaltar la innovación del programa de investigación de Juan Vicente Palerm en tres sentidos. Primero, porque aquella línea de recorridos de campo por localidades de España se llevaba a cabo en un área de estudios americanista. Él se encargó de que leyéramos obras en las que los americanistas habían prestado enorme atención a España, como Foster o Erasmus, sobre todo la obra de Arguedas, Las comunidades de España y del Perú (1968), que tanta influencia tendría en mi investigación doctoral. Segundo, porque en la docencia española en antropología dicha práctica docente y de investigación era más una excepción que una regla, dado que el hecho de que los alumnos hicieran prácticas de campo durante sus estudios era algo inusual. Además de los estudios referidos, cursé también la licenciatura en antropología social en la recién creada Facultad de Ciencias Políticas y Sociología. Tampoco en este otro departamento ni en esta facultad se promovían las prácticas de campo de los alumnos. Tercero, porque otros profesores no sólo no estimulaban esa línea de investigación y docencia sino que algunos expresaban explícitas reticencias sobre el particular. Recuerdo la negativa de uno de ellos cuando, como delegado de los alumnos, solicité apoyo para organizar trabajos de campo: "ustedes no están preparados, cuando acaben la licenciatura podrán hacerlo, ahora pueden dañar a los informantes".

La línea de práctica de campo desarrollada entre 1972 y 1975 gracias a los esfuerzos de Juan Vicente Palerm iba a contracorriente y no era fácil impulsarla, mientras en la UIA de México Ángel Palerm, Carmen Viqueira y colaboradores llevaban años organizándola con buenos resultados y se incluía como parte sustantiva del plan de estudios. En el "Prólogo" a Métodos de trabajo de campo, Juan Vicente Palerm afirma:

Hemos escogido este tema (el del trabajo de campo) en vista de las numerosas peticiones recibidas por parte de los estudiantes de antropología de la UCM. Esperamos que esta pequeña contribución incite el replanteamiento de nuevos problemas y la discusión dentro de la aulas, así como ayude a esclarecer algunos de los problemas acerca de los métodos de investigación (Palerm, J. V, 1971: i).

Se lee la conexión entre las investigaciones entonces en marcha - ese replanteamiento de nuevos problemas- con el aprendizaje de la metodología de campo - esa discusión dentro de las aulas-. En ese texto se hace la siguiente sugerencia:

Recomendamos, como complemento y para los estudiosos que deseen ampliar sobre el tema, la consulta de las Notes and Queries on Anthropology y del Outline of Cultural Materials, ambos publicados en su versión castellana por la Unión Panamericana (Washington, D. C., 1966 y 1960). ${ }^{2}$

${ }^{2}$ Subrayado en el original. 


\section{APRENDIENDO DIRECTAMENTE EN MÉXICO (1982- I 985)}

Cuando llegué a México, en octubre de 1982 con una beca predoctoral, ya conocía y practicaba en cierta medida varios elementos del trabajo de campo y su relación con la teoría y la formación de antropólogos, rasgos que se habían desarrollado bajo la influencia de Ángel Palerm. Había estado en México en 1980, decidido a concretar la dirección de mi tesis por parte del maestro, con quien había hablado el año anterior en Madrid, pero falleció el mismo año y su viuda Carmen Viqueira se hizo cargo de la dirección de mi tesis. Fue generosa, pues desde el primer momento me brindó su apoyo en alojamiento, contactos, orientaciones de todo tipo e incorporación a la UIA, en cuyo Departamento de Ciencias Sociales era profesora y del cual había sido directora. Con ella aprendería mucho, no sólo sobre antropología, sino también sobre lo que supone lo científico, sobre México y España y sobre la vida.

Durante aquella primera visita a México en 1980 hicimos un recorrido con Juan Vicente por el sur del valle de Toluca, donde el Departamento de Antropología de la UAM-I había instalado la Estación de Campo de San Lorenzo Huehuetitlán. En ese recorrido estuvimos por primera vez en San Nicolás Coatepec, una comunidad que dependía de la explotación del bosque comunal.

En la UIA había toda una tradición respecto de la incorporación del trabajo de campo a los estudios de antropología y en el currículum de los estudiantes de antropología mediante las prácticas de campo. La Universidad disponía desde 1968 de la Estación de Campo "José de Acosta" en la localidad de Tepetlaoxtoc, en la región de Texcoco, Acolhuacan, en el Estado de México, próxima al Distrito Federal. Por esa estación habían pasado y seguían pasando las nuevas generaciones de estudiantes de antropología. En ella se conserva actualmente un rico archivo de fichas - elaboradas y clasificadas según la Guía de Murdock- y materiales de campo. Nótese que me he referido a la "región de Texcoco", área de trabajo desde la estación como parte de esa concepción del entorno regional que conocía desde mis trabajos con Juan Vicente Palerm en la UCM y que sería clave en mis primeras investigaciones de campo de vuelta a España.

Aunque estaba adscrito a la UIA, mi trabajo de campo no sería desde la estación de campo de Texcoco sino en una región donde el tema de mi tesis, la propiedad comunal de la tierra y el régimen comunal agrario, fuera preponderante. Esa región fue el sur del valle de Toluca, también en el Estado de México, donde se llevaba a cabo un proyecto del Departamento de Antropología de la UAM-I, titulado "Movilidad geográfica y ocupacional de la mano de obra en el sur del valle de Toluca". Dicho proyecto sirvió para entrenar a estudiantes de licenciatura y produjo directamente, o fue apoyo clave, para varias tesis presentadas en universidades mexicanas y extranjeras, entre otras, las de R. M. Vázquez Mellado, J. C. Bolay y la mía. En esa área geográfica el Departamento de Antropología de la UAM-I disponía de una estación de campo, concretamente en la localidad de San Lorenzo Huehuetitlán, en el municipio de Santiago Tianguistenco.

El proyecto de la UAM-I había sido diseñado y puesto en operación por Juan Vicente Palerm. Al regresar a México, echó a andar el Proyecto "Agricultura y sociedad en El Bajío (1976-1979)", experiencia después de la cual lanzó el proyecto de Toluca. Cuando me incorporé a dicho proyecto (1982) el profesor Palerm ya no lo dirigía, pues se había marchado en 1981 a la UCSB. Carmen Viqueira y su hijo me pusieron en contacto con Laura González, profesora-investigadora de la UAM-I, quien había concluido su licenciatura en la UIA y cursaba la maestría en antropología social en esa universidad. Como estudiante, había aprendido y practicado el trabajo de campo al estilo de Palerm, y fue directora de la Estación de Campo de San Lorenzo Huehuetitlán durante los años que duró el Proyecto "Movilidad geográfica y ocupacional de la mano de obra en el sur del valle de Toluca", hasta fines de 1983. 
En dicha estación de campo, o "Casa de los Antropólogos de San Lorenzo", se formaron más de cuatro generaciones de antropólogos de la UAM-I, además de varios estudiantes de licenciatura y posgrado de la UIA y de otras universidades y centros de investigación mexicanos y europeos. Poco después del fallecimiento de Ángel Palerm (1980), el investigador Herbert Eling, de la Universidad de Texas en Austin, se trasladó a la estación de campo para colaborar con la profesora González y ambos recibieron y apoyaron a estudiantes provenientes de varias instituciones y lugares. Abraham Iszaevich de la uIA y Efraím Hernández Xolocotzi del Colegio de Posgraduados de Chapingo llevaron varias veces a sus estudiantes ahí para que el equipo de la UAM-I los preparara. Una larga serie de artículos y libros, así como de tesis de licenciatura y doctorado resultaron de los trabajos de campo realizados y/o apoyados en esa estación.

Nuestro encuentro fue afortunado ya que ella no sólo había aprendido teoría y método "a la palermiana" durante sus estudios en la UIA, sino que aplicaba y desarrollaba los principios, criterios o líneas de trabajo que he expuesto, como etnógrafa y trabajadora de campo. Laura lo hacía tanto como continuación de su propia investigación -que derivó en su tesis de licenciatura en la región de El Bajío, Guanajuato, bajo la dirección del profesor Juan Vicente Palerm - como desde el impulso de la nueva investigación que se llevaba a cabo en el valle de Toluca. Aprendí en aquel proyecto la importancia del enfoque regional, pues las investigaciones de campo sobre grupos étnicos, comunidades, mercados y estrategias campesinas ante el desarrollo del capitalismo, entre otros, se ubicaban en la historia y caracterización general de la región, y más concretamente del área situada al sur de la Gran Laguna de Lerma y de la carretera que une el Distrito Federal con Toluca, capital del Estado de México. En mi tesis comparé, tanto en la historia como en el presente, y siempre con énfasis en la diversidad ecológica, sistemas de tenencia de la tierra e instituciones comunales en la región pinariega en las provincias españolas de Burgos y Soria con el sur del valle de Toluca.

$\mathrm{Al}$ incorporarme al proyecto conocí el trabajo de supervisión que se hacía con los alumnos desplazados a zonas de esa región, cuyas prácticas primero y luego sus investigaciones se ubicaban en ésta o aquella comunidad. Observé cómo la maestra González recorría la zona, visitaba a los alumnos y supervisaba la marcha de su trabajo de campo. Esa experiencia, dentro de aquel enfoque o estilo, me permitió ejercitarme en varias técnicas de investigación. Las había estudiado en la carrera, pero no las había practicado, con excepción de las prácticas puntuales con Juan Vicente Palerm. Fue todo un hallazgo leer algunos de los diarios de campo y detenerme por un lado en los detalles - actividades diarias, reproducción de entrevistas, planes de trabajo, dibujos, esquemas, recortes, preguntas, reflexiones sobre uno mismo y su labor - y por otro en los comentarios anotados en la página izquierda por parte de la supervisora -indicaciones sobre la relevancia de lo descrito, observaciones para mejorar, cuestionamientos, posibles interpretaciones, relación de lo escrito con otros datos de otras comunidades, referencias bibliográficas-. Enseguida emprendí la tarea, compré varios cuadernos y comencé a escribir de esa forma: a) anotaba en la página derecha todo tipo de información relacionada con mi investigación; b) leía las anotaciones de mi supervisora, y c) redactaba yo mismo nuevos textos en la página izquierda al releer mi diario.

Además de los blocs de notas y de los diarios, aprendí la técnica de elaboración de fichas temáticas según el procedimiento usado en los recorridos, las prácticas y las investigaciones centradas en $\mathrm{Te}$ petlaoxtoc (UAM-I, 1976) para la clasificación de la información de campo. Seleccioné en la tabla de materias de la Guía los apartados necesarios en mi investigación sobre el régimen comunal agrario, en especial el apartado 42 dedicado a propiedad y dentro de él el subapartado 421, régimen de propiedad. Nada más conectar con Laura González y el Proyecto Toluca de la UAM-I participé en algunos 
recorridos de campo, algo habitual en etnografía y que Chambers (1985) incluye en su texto sobre métodos abreviados y rápidos para obtener información. Esas jornadas en Toluca no se reducían a meras caminatas con observación y anotaciones, sino que representaban la oportunidad de aprender a planificar los trayectos en función tanto de los conocimientos previos como de lo que se desea observar, de comentar lo observado y realizar paradas para la observación más sistemática de algo en concreto. En ese contexto quienes habían hecho recorridos con Ángel Palerm me contaron anécdotas de algunos ejercicios, como el de mirar una vivienda determinada durante un tiempo largo y contrastar lo que cada quien veía. De regreso a Madrid practiqué este ejercicio de campo con los estudiantes y comprobé cómo nuestra mirada es siempre parcial y la relevancia de los aportes del trabajo en equipo.

Había estudiado poco la entrevista etnográfica en la asignatura de métodos y técnicas dentro del plan de estudios en España. Me había sido de utilidad la publicación de Juan Vicente Palerm (1971) de un texto de 1953 de Benjamin Paul,3 "Técnicas de la entrevista y de las relaciones de campo". Lo que es más importante, había practicado la entrevista, pero no en la universidad, sino "por la libre", esto es, en la investigación de mi tesina de licenciatura sobre la propiedad e instituciones comunales en el Valle de Valdelaguna (Giménez, 1978). Pues bien, en la investigación de campo en México aprendí mucho sobre cómo hacer las entrevistas y quisiera hacer constar aquí la influencia que tuvo en mi trabajo un comentario que adjudicaban a Ángel Palerm. Me dijeron que el maestro insistía en dividir el tiempo de las entrevistas en tercios, algo así como "una hora para prepararla, una hora para llevarla a cabo, una hora para analizarla" - a todo ello hay que añadir el tiempo, siempre un tanto tedioso, de la transcripción-. Ciertamente, es algo sencillo y probablemente sabido, pero ¿cuántas veces no había

\footnotetext{
${ }^{3}$ Publicado originalmente con el título "Interview Techniques
} and Field Relationships" (Paul, 1953). preparado con detalle la entrevista y cuántas veces no la había analizado después? Lo relato solamente como un ejemplo más del trabajo de campo que vi hacer en México, e incluso como anécdota.

En lo que corresponde a los estudios de caso de familias campesinas se me informó de los estudiantes en prácticas y/o los investigadores que los estaban llevando a cabo. En este punto también me beneficié de los aportes de Laura González, quien había analizado familias campesinas de manera pormenorizada para su tesis sobre las estrategias del campesinado en Valle de Santiago, Guanajuato. Los investigadores habían incluido en sus notas, en los diarios y en las fichas aspectos relacionados con el tamaño y composición de grupos domésticos, su trayectoria o historia, sus recursos en tierra, ganado, construcciones, etc., la división del trabajo en el seno de la familia y las actividades de cada miembro - había fichas por cada uno-, las relaciones internas y externas, y demás. El estudio de una determinada familia podía completarse con una historia de vida o una biografía - del padre o de la madre, por ejemplo- o estar incluido en una genealogía más amplia. Aprendí a combinar técnicas de investigación respecto de determinados informantes de la siguiente forma: individuo-historia de vida, familia-estudio de caso y parentela-genealogía, material informativo que mucho después vendría a sumarse al network o red de relaciones. Esta estrategia de investigación, aprendida en el campo y ligada a las teorías sobre campesinado, grupo doméstico, parentesco, etc., me sería muy útil para diseñar las investigaciones que emprendería en España.

Recuerdo las sesiones y las horas dedicadas en la Estación de Campo de San Lorenzo Huehuetitlán a analizar las genealogías elaboradas y a iniciarme en este arte con mi propia familia de la mano de Laura González. Ángel Palerm había influido años atrás en el uso intensivo de la técnica de la genealogía, en especial por su valoración en el sentido de las aportaciones de William H. R. Rivers (1864-1922), sobre todo su artículo "El método genealógico de investigación antropológica", publicado originalmente en The Sociological Review en 1910. Ángel Palerm afirmaba: 
El artículo es uno de los pocos que con justicia pueden calificarse de seminales en la historia de la antropología, aunque resulta obvio que las técnicas de registro y análisis que elaboró han sido modificadas y perfeccionadas desde entonces (Palerm, Á., 1977: 152).

Desde la Estación de Campo de San Lorenzo Huehuetitlán me trasladé a la localidad de San Nicolás Coatepec de las Bateas, en el municipio de Santiago Tianguistenco, donde desarrollé mi estancia de larga duración y la investigación para mi tesis doctoral. Fue un privilegio contar con el marco general de un proyecto regional y con la supervisión de una experimentada colega, contrastar mis informaciones con las de otros investigadores y con un amplio repertorio de procedimientos que iba aprendiendo. Todo ello y en su conjunto la experiencia vivida en instituciones y con equipos de trabajo mexicanos fueron de gran inspiración y utilidad en el regreso a España y en la incorporación a la universidad. Como colofón de aquella época de aprendizaje intensivo pude disfrutar para la redacción de la tesis de una fructífera estancia en el Departamento de Antropología de UCsB, invitado por Juan Vicente Palerm.

\section{APLICACIÓN Y TRANSMISIÓN DEL LEGADO TRAS LA VUELTA A ESPAÑA EN 1985}

Volví a España a principios de octubre de 1985 como becario de reincorporación a la UAM. Aquel programa de becas del Ministerio de Educación y Ciencia estaba orientado a facilitar la vuelta de quienes habíamos ido fuera de España con becas predoctorales. Había hecho contactos con mis profesores de la UCM para ingresar a alguno de los dos departamentos donde estudié antropología y obtuve licenciatura -el Departamento de Antropología y Etnología de América de la Facultad de Geografía e Historia y el Departamento de Antropología Social de la Facultad de Ciencias Políticas y Sociología-, pero mi solicitud oral no prosperó en ninguno. Afortunadamente, pude adscribir al Departamento de Filosofía de la UAM mi beca de reincorporación, aunque no había estudios de licenciatura en antropología sino un grupo de antropólogos que trabajaba ahí desde hacía años.

Llegué a España y a la UAM de manera un tanto precipitada, pues acababa de suceder el terremoto en México y tuve que dedicar las últimas semanas de mi estancia ahí a localizar a amigos y compadres, y apoyar en lo posible. Recibí el encargo de varias clases y grupos dentro del plan docente del curso 1985-1986. En aquellas primeras clases de antropología comencé a aplicar contenidos clave aprendidos en México. En lo teórico incorporé, al configurar los programas de clases, la concepción triple de Ángel Palerm acerca de la antropología: como tradición cultural, como ciencia social y como profesión (Palerm, Á., 1967 y 1974). Integré esta idea sugerente acerca de la naturaleza de la antropología como eje vertebrador de la memoria docente presentada dos años más tarde al concurso de oposición para la plaza de profesor titular en antropología. Desde entonces, en los cursos y seminarios he aprovechado y dado a conocer las aportaciones de Ángel Palerm sobre los precursores del mundo clásico, la era de los descubrimientos, las diferentes colonizaciones y la era de las revoluciones según su Historia de la etnología (1974). Asimismo, como profesor de cursos de introducción a la antropología he utilizado otros enfoques y aportes de la obra de Palerm.

En lo metodológico y técnico incluí en la programación de los cursos ejercicios prácticos de observación en la ciudad de Madrid. En cada curso y con cada grupo de estudiantes hicimos recorridos urbanos y visitas planificadas, por ejemplo, por El Rastro madrileño: extenso y heterogéneo mercadillo de fin de semana en la Ribera de Curtidores, límite este del antiguo, céntrico, popular y castizo barrio de Lavapiés. ${ }^{4}$ Por otra parte, los alumnos elaboraban en casa la respectiva genealogía de su familia. En el aula aprendían los fundamentos de esta técnica y

\footnotetext{
${ }^{4}$ Barrio que luego estudiaría mediante trabajo de campo en 1996 y 1997, y como reestudio de caso en 2006 y 2007.
} 
analizábamos los casos que traía de Coatepec. Luego exponían sus trabajos y se analizaban colectivamente en clase. La realización de estos ejercicios formaba parte de la evaluación del curso.

\section{EL SEMINARIO DE INVESTIGACIÓN ANTROPOLÓGICA: EL PROYECTO SIERRA NORTE DE MADRID Y OTROS PROYECTOS (1985-1989)}

Aparte de esa influencia general de la obra de Palerm en mis cursos, seminarios y charlas, quiero relatar en particular la aplicación de lo aprendido en México y del enfoque palermiano sobre la interacción de teoría, trabajo de campo y formación de antropólogos en la creación y desarrollo del Seminario de Investigación Antropológica de la UAM (SIA-UAM), como espacio universitario para realizar trabajo ligado a proyectos de investigación y como parte de la formación de los jóvenes antropólogos. Los dos proyectos iniciales que impulsé en paralelo desde el SIA-UAM fueron una recopilación bibliográfica de los estudios de antropología social sobre la España rural desde 1946 (Giménez, 1986) y el proyecto de investigación en una región montañosa y ganadera situada al norte de la capital.

Desde que me incorporé a la UAM tenía la voluntad de llevar a los estudiantes al campo, por eso hicimos los primeros recorridos por la Sierra Norte de Madrid en el primer trimestre de 1986. Se trata de la zona más desfavorecida de la Sierra de Guadarrama, ubicada al norte y noreste de la provincia de Madrid. ${ }^{5}$ Esta región es una zona ideal para prácticas de campo por su cercanía a Madrid — de 60 a 100 kilómetros según el punto de la Sierra Norte que consideremos-, por su considerable extensión -1400 kilómetros cuadrados- y por su notable diversidad de subáreas ecológicas, influencias históricas, comarcas y localidades -42 municipios- A la Sierra

\footnotetext{
${ }^{5}$ Provincia de Madrid que desde 1980 tiene el estatuto de Comunidad Autónoma de Madrid.
}

Norte se le llama la "sierra pobre" por el contraste con la "sierra rica", área localizada al noroeste de la capital y correspondiente a San Lorenzo de El Escorial, Villalba, Los Molinos y otras.

Se integraron a dichas actividades etnográficas tanto licenciados recientes - Juan Carlos Gimeno, Montserrat Hurtado y Pilar Monreal- que hacían sus trabajos de campo iniciales en la ciudad de Madrid o en su periferia como un nutrido grupo de estudiantes de los cursos cuarto y quinto de la licenciatura de filosofía. Había un déficit claro de trabajo de campo, sobre todo en equipo, planificado y ligado a los estudios. Debo mencionar aquí la gran aportación de Juan Carlos Gimeno, Montserrat Hurtado y Pilar Monreal quienes, además de servir de puente con los estudiantes que aún no terminaban sus estudios de licenciatura, actuaron en todo momento como monitores y se hicieron cargo de la coordinación del Seminario y del proyecto durante dos cursos cuando hube de marcharme con una beca posdoctoral a la UCSB. Asimismo, fue muy valiosa la contribución de Paloma Gómez Crespo, también recién licenciada, que jugó un papel clave en el apoyo al Proyecto Sierra Norte y en la recopilación bibliográfica.

Nuestra actividad no consistió meramente en un conjunto de visitas, prácticas o recorridos, sino que, como en México, las estancias progresivas en el campo formaron parte de un proyecto de investigación formalizado en julio de 1987. El proyecto tenía por título "Transformaciones socioeconómicas en la Sierra Norte de Madrid (1900-1987). Estudio del cambio en las relaciones campo-ciudad" (Giménez, 1987) y estaba centrado en el problema del cambio sociocultural, que tanto enfatizó Ángel Palerm, y en los estudios campesinos con enfoque articulacionista referido. En la "Introducción" al proyecto se ubican así sus antecedentes:

En aquellos recorridos surgió el interés por el área con base en dos consideraciones, una de orden teórico y otra de orden práctico. Interesó por la diversidad de fenómenos nuevos en una zona que revelaba una profunda alteración: embalses, urbanizaciones, carretera 


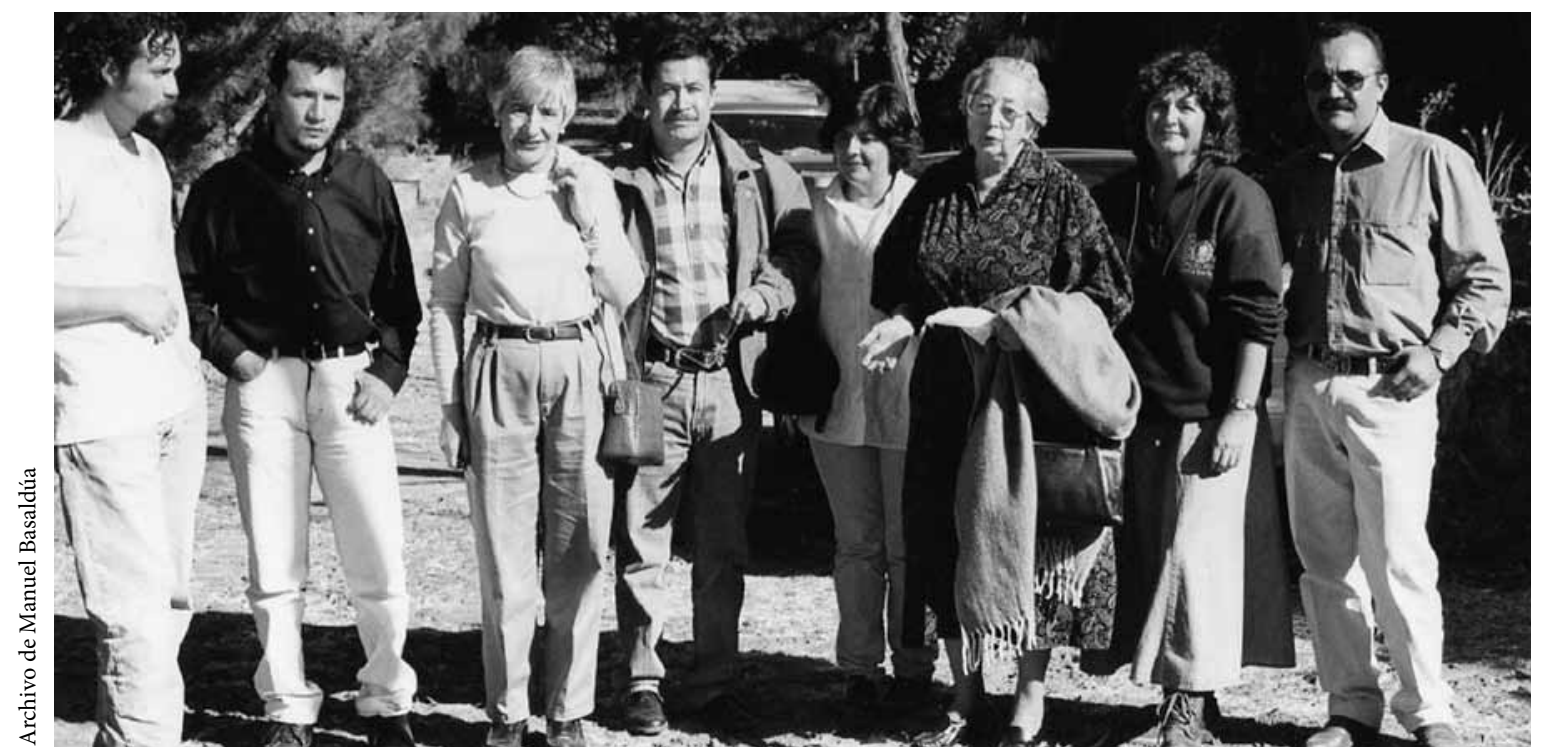

La Ordeña, municipio de Salamanca, Guanajuato, 2000. De izquierda a derecha: dos estudiantes de Laura González, Marisol Pérez Lizaur, Manuel Basaldúa, Martha Otilia Olvera Estrada, Carmen Viqueira, Laura González y Gaspar Real Cabello.

nacional, reconversión ganadera, fuerte presencia de la administración autonómica, etc. Por otra parte, la cercanía de la Sierra Norte de Madrid ayudaba a la realización de trabajo de campo, habida cuenta de la falta de presupuesto y de tiempo académico. Era preciso escoger una zona de fácil acceso y que permitiera aprovechar fiestas, vacaciones y fines de semana para los primeros recorridos (Giménez, 1987: 1). ${ }^{6}$

Se refleja aquí el interés por los problemas de cambio sociocultural, eje temático del proyecto, así como la ligazón de investigación y acción formativa mediante el trabajo de campo:

Tomada la decisión, el equipo de investigación fue preparándose mediante el estudio de textos metodológicos, de monografías antropológicas sobre el campo español y de obras claves de la teoría del campesinado (Giménez, 1987: 1).

Aquello fue como revivir, con las diferencias de contexto y maduración, lo vivido en el Seminario de Estudios Campesinos de la UCM, o continuar con lo

\footnotetext{
${ }^{6}$ Énfasis añadido.
}

aprendido en el proyecto del valle de Toluca. No es aquí el lugar para detallar ese proyecto, sus resultados y los aportes de cada cual, pero sí para dejar constancia de cuatro aspectos constitutivos de aquella experiencia universitaria en equipo, docente y de investigación: dos de esos elementos están relacionados con la teoría antropológica y otros dos con el método y el repertorio de técnicas de investigación.

\section{Teoría del cambio sociocultural y enfoque de Ángel Palerm}

En primer lugar, debo señalar la clara influencia de algunos énfasis teóricos de Ángel Palerm en el diseño y marco conceptual del proyecto. Por una parte, en el planteamiento del problema central de investigación, esto es, "la naturaleza y las causas del cambio económico y social que en dicha área venía produciéndose" (Giménez, 1987: 23). Así lo formulamos en el proyecto:

Plantear dicha problemática como nudo gordiano del estudio de esta región ha venido dado por tres 
razones. En primer lugar el tratarse de un área de profundas transformaciones [...] En segundo lugar [...] por la preocupación e incertidumbre existente -y no sólo ni principalmente en nosotros - respecto al futuro inmediato de la sociedad rural española y de la economía agraria. [...] La tercera razón [...] es el convencimiento de la extraordinaria importancia de la evolución como núcleo de la problemática del cambio sociocultural. Para autores como Ángel Palerm la evolución constituye el tema central de la antropología (Giménez, 1987: 24).

Y sigue una larga cita al respecto de su Teoría etnológica. Proseguíamos así:

Nuestro siguiente paso es concretar para la Sierra Norte de Madrid la problemática del cambio. También Palerm nos dará una pauta para ello cuando afirma: "Cualquiera que sea el enfoque conceptual adaptado (respecto a la cuestión del cambio sociocultural), es indudable que una teoría dinámica debe estar en condiciones de facilitar ciertas respuestas a algunos problemas esenciales. Probablemente, el primero de ellos consiste en identificar y medir la magnitud de los cambios socioculturales. El segundo problema es el de indicar las fuerzas responsables de los cambios. El tercero consiste en determinar la dirección o las tendencias de los cambios. El cuarto y último de los problemas esenciales de la teoría dinámica, es el de anticipar o pronosticar las posibilidades de las formas sociales emergentes (Giménez, 1987: 24-25).

A partir de ahí lo que hicimos en el Proyecto Sierra Norte fue aceptar esos cuestionamientos, fundir el tercer interrogante con el cuarto y añadir la cuestión de los mecanismos y repercusión de los cambios, con lo que el problema central de investigación se concretó en éstos: 1) ¿Qué ha cambiado y cuáles han sido y están siendo los niveles del cambio? (o problema de la naturaleza y dimensiones del cambio); 2) ¿Por qué ha cambiado? (o problema de los factores exógenos y endógenos del cambio); 3) ¿Cómo ha cambiado? (o problema de los mecanismos del cambio), y 4) ¿Hacia qué cambia? (o problema de las tendencias del cambio).

\section{Trabajo de campo y discusión de la teoría}

Un segundo rasgo de la experiencia fue el hecho de que los participantes combinaron, durante aquellos cuatro años del Proyecto Sierra Norte, la lectura y la discusión de la teoría y la metodología con la investigación de campo. No puedo tampoco detallar aquí este aspecto, sólo mencionaré algunos textos clave a partir del propio documento del proyecto con la doble finalidad de mostrar qué lecturas fueron revisadas y discutidas en los seminarios en la Universidad y profundizadas en la experiencia de campo y, por otro lado, cómo en buena parte de esas lecturas está clara la influencia de la trayectoria en España y México referida en los apartados anteriores. Tras el planteamiento de problemas expuesto, se describió el enfoque teórico con base en "los estudios de cambio sociocultural", los cuales se sintetizaron "para nuestro proyecto Sierra Norte de Madrid" en 16 puntos. Entre ellos figuraban:

- que el cambio es adaptación, conflicto y evolución, cito aquí la expresión de Steward de "secuencia temporal de formas", lo que llevó a leer a algunos ecólogos culturales, teóricos del conflicto y sobre todo a evolucionistas multilineales;

- que el cambio tiene una dimensión sincrónica o estructural-funcional y una diacrónica o temporal, como insistía una y otra vez Ángel Palerm en diferentes escritos - a partir de, entre otros, la lectura de Saussure-, aspecto que fue sostenido y discutido con textos de Leach o Radcliffe-Brown, así como de la lectura de Braudel para tener en cuenta la larga, media y corta duraciones;

- que no todas las instituciones tienen el mismo peso en la sociedad, de modo que aprovecho la idea de Palerm sobre "instituciones estratégicas" y reconozco en este punto el "carácter decisivo de las fuerzas productivas y de las relaciones sociales de producción" y su relación con la dimensión ideológica, con la consiguiente lectura de 
Marx y de ensayos marxianos dispares de Palerm, Krader o Harris;

- que las sociedades rurales pueden ser entendidas como "segmentos sociales relacionados asimétricamente con la sociedad mayor", respecto de lo cual se recurre a la consulta y discusión de textos - plurales en su enfoque- de Redfield, Foster, Banfield, Shanin, Wolf, Warman y otros;

- que "el estudio del cambio rural puede contemplarse enfatizando a) los cambios locales y su referencia al contexto global; b) los cambios extralocales y su repercusión en la sociedad rural, y c) los cambios en las relaciones económicas, políticas y culturales entre la sociedad rural y la sociedad mayor" (Giménez, 1987: 37-38), lo que incorpora como elementos conceptuales de referencia en el Proyecto la lectura de Chayanov y de la polémica entre sustantivismo y formalismo; la teoría de la dependencia (Frank, Amin) y del moderno sistema mundial (Wallerstein, Palerm, Mintz, Wolf); o los debates sobre modos de producción y su articulación (como modelos propuestos por Ángel Palerm a partir de la fórmula M-D-M - mercancía, dinero, mercancía-de Marx.

No he querido decir que todos los textos fueran leídos y discutidos por todos los participantes en el proyecto, sino que aquella experiencia formativa de trabajo de campo formó parte de un proyecto de investigación, en buena parte inspirado en el énfasis teórico de Ángel Palerm, y que los licenciados y estudiantes de los últimos cursos leyeron una serie de textos y los discutieron en relación con el proyecto y su etnografía.

\section{Metodología de campo y trabajo en equipo}

En cuestiones de método, quiero subrayar que la forma de trabajo también partía de lo aprendido con Juan Vicente Palerm, concretado y desarrollado en mi estancia en México. El proyecto tuvo tres fases: la inicial de recorridos y estancias breves, la intermedia de estudios de comunidad y la final de elaboración de informes temáticos, cuatro de los cuales se presentaron como tesis de licenciatura. Después de la primera, los estudiantes se distribuyeron en distintas localidades para realizar estancias progresivas en cuanto a duración. Entre tanto hacíamos reuniones ya fuera con cada estudiante en su localidad o todos juntos, por lo general en la casa del pueblo de Gascones, donde desarrollaba su trabajo de campo uno de los estudiantes y con cuyos dueños teníamos una magnífica relación. En aquella casa los alumnos se iniciaron en el arte de compartir observaciones, discutir nociones de teoría o método o leer partes de los diarios y comentarlos juntos.

Un paso cualitativo en el proyecto fue la elaboración de los "Informes de comunidad", para lo que se estructuró una "Guía temática para la redacción de las monografías", esquema que adaptaba las pautas de las monografías etnográficas a los objetivos del proyecto y las características de la región. Durante 1987 se completaron siete reportes de comunidad: Díaz (1987), Dochao (1987), Jabardo (1987), Pérez del Olmo (1987), Ramírez (1987), San Bruno (1987) y Suanzes (1987). Tras esa fase se fueron profundizando diversos estudios temáticos. Cada investigador definió un tema y aprovechó el conjunto de los materiales recogidos así como su trabajo de campo en una comunidad, redactó un "Informe" sobre esa cuestión y completó la indagación de campo que fuera precisa. En un número monográfico que la revista Alfoz dedicó en 1991 a la Sierra Norte, se publicaron tres síntesis de esos trabajos: cambios territoriales (Hurtado, Díaz y Ramírez, 1991), evolución económica (Gimeno, Gómez y Dochao, 1991) y tejido social (Monreal et al., 1991). Las temáticas del turismo rural (Dochao, 1986), migraciones (Jabardo, 1990), ganadería (Pérez del Olmo, 1989) y tenencia de la tierra (Ramírez, 1990) fueron objeto de tesis de licenciatura, uno de los principales productos del proyecto. 


\section{Elaboración y aplicación colectiva de las técnicas de investigación}

Un cuarto elemento definidor de la experiencia formativa y de investigación del Proyecto Sierra Norte comprende el despliegue y la aplicación de un amplio repertorio de procedimientos técnicos de registro, clasificación y análisis de la información, aplicados de manera individual y compartidos colectivamente. Un dispositivo central en lo metodológico y técnico fue el conjunto de fichas temáticas para el registro de la información, las cuales dan constancia de algunas de las unidades de análisis especificadas en el proyecto: "Fichas de contenidos", "Fichas de unidades domésticas", "Fichas de explotaciones ganaderas", "Ficha de patrimonio rústico", "Ficha de turismo rural", "Ficha de comercio" y "Fichas de localidad". Estos protocolos de registro y clasificación de la información formaron parte de los sucesivos anexos del documento central del proyecto.

En ese proceso de creación de procedimientos unificados de recolección y clasificación de la información se refleja claramente lo aprendido en México y la tradición de fichas tanto en las actividades de la UIA en Tepetlaoxtoc como en el proyecto de la UAM-I en Toluca y El Bajío. Ahora bien, también y sobre todo se hace patente la creatividad del propio proyecto, dado que esos protocolos técnicos del Proyecto Sierra Norte fueron discutidos con y enriquecidos por los coordinadores y estudiantes, y estaban lógicamente adaptados a la naturaleza del proyecto y a las particularidades del área de estudio. Suponían, como no podía ser de otra manera, un desarrollo de los instrumentos con los que me había dotado en México. Quiero enfatizar aquí lo que hay de hilo conductor y transmisión generacional entre experiencias iniciadas en México a finales de la década de 1970, desarrollos varios posteriores y lo que hemos hecho de forma diversa y creativa en diferentes lugares de México, California o España, iniciativas inspiradas en un enfoque cuya piedra angular remite a Ángel Palerm.

Para dar idea del detalle de esos instrumentos de investigación —como aprendí y ejercité en Toluca y en otras experiencias de campo en México- comento brevemente tres de ellos. Las fichas de contenido fueron la unidad menor, pues en ellas se depositaban datos concretos e información propiamente dicha con informante o documento, fecha, lugar, investigador y código de clasificación según la Guía de Murdock y su aplicación en el proyecto. Las fichas de unidades domésticas constaban de una serie de ocho plantillas, cada una con su detalle correspondiente: 1) ficha resumen, 2) evolución histórica de la familia, 3) historia de vida de al menos uno de los miembros, 4) movimientos migratorios familiares, 5) relaciones socioeconómicas familiares, 6) presupuesto familiar, 7) vivienda y dependencias agrícolas, y 8) evolución económica y ocupacional. Las fichas de localidad suponían asimismo un paquete de otras ocho subfichas y sus campos de información respectivos: 1) localización y datos básicos, 2) demografía, 3) usos de suelo, 4) ganadería y maquinarias, 5) actividad económica del pueblo, 6) equipamiento y servicios, 7) asociaciones y administración, y 8) ciclo anual festivo. Con esos dispositivos o esquemas de registro y clasificación se elaboraron cientos de informaciones particulares, decenas de estudios de caso y una serie de reportes sobre la localidad, los cuales forman parte del Fondo Documental del Proyecto y estaban al alcance de cualquiera para su uso "transversal" para éste o aquel tema. A todo ello hay que añadir las genealogías.

\section{El Proyecto de Colonización Agraria}

Antes de finalizar este relato sobre el SIA-UAM, su grupo y actividades, debo mencionar el otro proyecto de investigación que desarrollamos desde ese espacio: el Proyecto de Colonización Agraria, el cual formó parte de un programa amplio de investigación sobre la "Historia y evolución de la colonización agraria en España”. La colonización agraria fue uno de los procesos más intensos de transformación del campo español, llevado a cabo desde la 
posguerra e implementado casi siempre mediante la construcción de presas y nueva agricultura de regadío en decenas de zonas de colonización y con la construcción de más de 300 poblados de colonización. Se trató de un encargo del Ministerio de Agricultura, Pesca y Alimentación, a partir del impulso de Cristóbal Gómez Benito, sociólogo rural, estudioso de Costa y por entonces director de Publicaciones del Ministerio. En aquel macroproyecto sobre la colonización agraria participaron decenas de especialistas de distintas disciplinas, coordinados por el arquitecto A. Villanueva. A nosotros nos correspondió el estudio sociológico y antropológico, y realizamos trabajo de campo en tres zonas de colonización: Bardenas Reales, Zaragoza; Montijo, Badajoz, y Bajo Guadalquivir, Sevilla. Para efectos de este artículo y del relato de la extensión a España del enfoque palermiano sobre teoría, trabajo de campo y formación, sólo destacaré los siguientes aspectos de este segundo proyecto de investigación: ${ }^{7}$

- El aprovechamiento de la intensa y valiosa aportación de Ángel Palerm sobre todo lo relativo a agricultura, regadío y sociedad hidráulica, tema esencial en su obra.

- Participación en el proyecto de jóvenes investigadores que continuaron su formación en ese nuevo marco. En la investigación de campo intervinieron, junto a otros investigadores, Gimeno, Hurtado y Monreal, quienes ya me habían acompañado en el Proyecto Sierra Norte, lo cual fue clave para aprovechar lo hecho y darle continuidad.

- De nuevo la relevancia del enfoque regional para contextualizar y comparar las zonas estudiadas.

- Elaboración de más de un centenar de estudios de caso de explotaciones agrarias en los poblados, siguiendo las pautas mencionadas.

\footnotetext{
${ }^{7}$ Para más detalles, véanse Giménez (1992) y Giménez y Sánchez (1994).
}

\section{A MODO DE CONCLUSIÓN}

El producto más relevante de aquella experiencia formativa y de investigación, ligada históricamente a lo que hicieron muchos maestros e investigadores en México y a la figura y presencia intelectual de Ángel Palerm, fue la formación original, innovadora, colectiva y creativa de todo un elenco de jóvenes antropólogos. Varios de ellos - por orden alfabético, Juan Carlos Gimeno, Paloma Gómez Crespo, Mercedes Jabardo, Pilar Monreal y Ángeles Ramírezson hoy profesores universitarios de antropología. En sus clases, programas y elaboraciones sigue presente, de forma totalmente plural, aquel enfoque compartido. Miembros del Seminario fueron en los años siguientes a México, por ejemplo, Fernando Pérez del Olmo para hacer su tesis o Juan Carlos Gimeno y Pilar Monreal como doctores y profesores, lo que intensifica los vínculos y facilita nuevos aportes.

Por mi parte, fui a California en 1987 a hacer trabajo de campo en el Valle de San Joaquín e investigar las consecuencias socioculturales de la agricultura de regadío y compararlas con lo estudiado en España, todo como parte del proyecto de Juan Vicente Palerm sobre nuevas comunidades rurales. Durante esa nueva experiencia pude observar cómo Juan Vicente, con quien comencé esta narración, desplegaba todo un programa de investigaciones de campo con doctorandos y estudiantes de grado con el mismo enfoque de investigación y formativo al que me había enganchado muchos años atrás. De vuelta en España, en 1989, configuré el Proyecto "Inmigración extranjera en España: estudios y asesoramiento", que dio lugar desde 1994 al Programa Migración y Multiculturalidad (PMM), de carácter teórico y aplicado. ${ }^{8}$ A su vez, el PMм fue el núcleo principal del impulso mancomunado por parte de

\footnotetext{
${ }^{8} \mathrm{Al}$ PMM, en el que han participado numerosos investigadores y profesionales de la intervención, se sumó desde sus comienzos la profesora Liliana Suárez, quien en esas fechas recién regresaba de la Universidad de Stanford y aportó, entre otras muchas cosas, su intensa vinculación con México.
} 
varios profesores y equipos para crear en 2006 el Instituto Universitario de Investigación en Migraciones, Etnicidad y Desarrollo Social (IMEDEs) que propusimos al rector, sin fortuna, denominar "Instituto Ángel Palerm".

No evoco este relato como una simple memoria de actividades sino para mostrar cómo determinadas semillas fructifican, brotan y van uniendo en el tiempo influencias y avances. Un botón de muestra y de cierre. Desde nuestro Departamento de Antropología y Pensamiento Filosófico Español de la UAM, que creó el Aula "Ángel Palerm", y con la colaboración del IMEDES, estamos participando en una red española de instituciones universitarias que sirva como extensión de la Cátedra “Ángel Palerm”, conformada por cuatro instituciones mexicanas: la UIA, la UAM-I, el CIESAs y El Colegio de Michoacán (Colmich). Obsérvese que en tres de esas instituciones el maestro Ángel Palerm jugó un papel absolutamente fundador e impulsor de la interacción entre historia de la teoría, el trabajo de campo y la formación de antropólogos.

\section{BIBLIOGRAFÍA}

Arguedas, José María, 1968, Las comunidades de España y del Perú, Universidad Nacional Mayor de San Marcos, Lima.

Chambers, Robert, 1995, "Métodos abreviados y participativos a fin de obtener información social para los proyectos", en Michael Cernea (coord.), Primero la gente. Variables sociológicas en el desarrollo rural, Fondo de Cultura Económica, México, pp. 587-611.

Contreras, Jesús, Juan José Pujadas e Ignasi Terradas, 2000, "Ángel Palerm y los antropólogos españoles del 68", en Virginia García Acosta (coord.), La diversidad intelectual. Ángel Palerm in memoriam, Centro de Investigaciones y Estudios Superiores en Antropología Social, México, pp. 43-62.

Díaz Dancausa, M., 1987, "Informe de comunidad: la Puebla de la Sierra", Seminario de Investigación Antropológica, Universidad Autónoma de Madrid, Madrid.

Dochao, Andrés, 1986, Ocio, ecología y juventud, Dirección General de Juventud (Cuadernos de Juventud), Madrid.

_, 1987 , "Informe de comunidad: Rascaría", Seminario de Investigación Antropológica, Universidad Autónoma de Madrid, Madrid.
Fábregas, Andrés, 2000, “Ángel Palerm. Un maestro inolvidable”, en Virginia García Acosta (coord.), La diversidad intelectual. Ángel Palerm in memoriam, Centro de Investigaciones y Estudios Superiores en Antropología Social, México, pp. 211-222.

,- 2010 , Configuraciones regionales mexicanas. Un planteamiento antropológico, t. I, Gobierno del Estado de Tabasco, Centro de Estudios Superiores de México y Centroamérica-Universidad de Ciencias y Artes de Chiapas, Tuxtla Gutiérrez.

García Acosta, Virginia (coord.), 2000, La diversidad intelectual. Ángel Palerm in memoriam, Centro de Investigaciones y Estudios Superiores en Antropología Social, México.

Giménez, Carlos, 1978, "Estudio antropológico de las instituciones y usos comunales en el Valle de Valdelaguna (Burgos)", Gabinete de Estudios y Asesoramiento, Madrid, mimeo.

(coord.), 1986, “Trabajos de campo en antropología rural en España (1946-1986)”, Seminario de Investigación Antropológica, Universidad Autónoma de Madrid, Madrid.

— 1987 , "Proyecto general de investigación: transformaciones económicas y sociales en la Sierra Norte de Madrid (1900-1987). Estudio del cambio en las relaciones campo-ciudad", Seminario de Investigación Antropológica, Universidad Autónoma de Madrid, Madrid.

— 1991, Permanencia y funcionalidad del régimen comunal agrario en España y México, Ministerio de Agricultura, Pesca y Alimentación, Madrid.

_, 1992, "Transformaciones económicas y sociales en la Sierra Norte de Madrid (1900-1990)", en Alfoz, núm. 83 , pp. 39-46.

_ 1994 , "Agua y poder. Breve historia de las ideas en torno a la irrigación. Democracia y despotismo", en Juan Romero y Carlos Giménez (eds.), Regadíos y estructuras de poder, Instituto de Cultura "Juan Gil Albert”, Diputación de Alicante, Alicante, pp. 15-40.

- y Luciano Sánchez (coords.), 1994, Unidad y diversidad de la colonización agraria. Perspectiva comparada del desarrollo de las zonas regables, Ministerio para las Administraciones Públicas, Ministerio de Agricultura, Pesca y Alimentación, Ministerio de Obras Públicas, Transporte y Medio Ambiente, Madrid.

Gimeno, Juan Carlos, Paloma Gómez Crespo y Andrés Dochao, 1991, "La evolución económica en la Sierra Norte de Madrid: adaptación o retraso", en Alfoz, núm. 83, pp. 62-66.

González, Laura, 1991, Respuesta campesina a la Revolución Verde en el Bajío, Universidad Iberoamericana, México. 
Guía para la clasificación de los datos culturales, 1976, Universidad Autónoma Metropolitana-Iztapalapa, México.

Hurtado, Montserrat, María Díaz y Ángeles Ramírez, 1991, "Los cambios territoriales en la Sierra Norte de Madrid: el pesado influjo de la capital", en Alfoz, núm. 83 , pp. 56-61.

Jabardo, Mercedes, 1987, "Informe de comunidad: Navarredonda”, Seminario de Investigación Antropológica, Universidad Autónoma de Madrid, Madrid.

-, 1990, "Las relaciones campo ciudad a través de los emigrados: Madrid y su Sierra Norte", tesis de licenciatura, Departamento de Sociología y Antropología Social-Universidad Autónoma de Madrid, Madrid.

Kearney, Michael, 1986, "From the Invisible Hand to Visible Feet. Anthropological Studies of Migration and Development", en Annual Review of Anthropology, núm. 15.

Krader, Lawrence, 1972, The Ethnological Notebooks of Karl Marx, Van Gorcum, Assen.

Kropotkin, Piotr, 1970, El apoyo mutuo, zyx, Madrid.

Marx, Carlos, 1972, “Cuadernos etnológicos”, en Lawrence Krader, The Ethnological Notebooks of Karl Marx, Van Gorcum, Assen.

- 1978, Formaciones económicas precapitalistas, Ediciones de Pasado y Presente, México.

Melville, Roberto, 1994, "La participación ciudadana en la construcción de presas en el Valle del Tennessee", en Juan Romero y Carlos Giménez (eds.), Regadíos y estructuras de poder, Instituto de Cultura "Juan Gil Albert”, Diputación de Alicante, Alicante, pp. 101-120.

_ 2000, "Ángel Palerm y la investigación antropológica en el México de hoy", en Virginia García Acosta (coord.), La diversidad intelectual. Ángel Palerm in memoriam, Centro de Investigaciones y Estudios Superiores en Antropología Social, México, pp. 23-24.

Monreal, Pilar et al., 1991, "El tejido social serrano", en Alfoz, núm. 83, pp. 67-72.

Narotsky, Susana, 2002, “The Political Economy of Political Economy in Spanish Anthropology”, en Winnie Lem y Belinda Leach (eds.), Culture, Economy and Power. Anthropology as Critique, Anthropology as Praxis, State University of New York Press, Nueva York.

Ortí, Alfonso, 1994, "Política hidráulica y emancipación campesina en el discurso político del populismo rural español (entre las dos repúblicas contemporáneas)", en Juan Romero y Carlos Giménez (eds.), Regadíos y estructuras de poder, Instituto de Cultura "Juan Gil Albert”, Diputación de Alicante, Alicante, pp. 241-267.

Palerm, Ángel, 1967, Introducción a la teoría etnológica, Instituto de Ciencias Sociales-Universidad Iberoamericana (Colección del Estudiante de Ciencias Sociales, núm. 1), México.
, 1974, Historia de la etnología, t. I: Los precursores, Centro de Investigaciones y Estudios Superiores en Antropología Social, México.

, 1977, "Rivers. El nacimiento de la antropología social”, en Ángel Palerm, Historia de la etnología, t. III: Tylor y los profesionales británicos, Centro de Investigaciones y Estudios Superiores en Antropología Social, México, pp. 149-167.

, 1980, Antropología y marxismo, Nueva Imagen, México.

_ y Juan Vicente Palerm, 1976, “Introducción”, en Guía para la clasificación de los datos culturales, Universidad Autónoma Metropolitana-Iztapalapa, México.

Palerm Viqueira, Jacinta (ed.), 1992, Guía y lecturas para una primera práctica de campo, Universidad Autónoma de Querétaro, Querétaro.

Palerm Viqueira, Juan Vicente, 1971, “Prólogo”, en Métodos de trabajo de campo, Departamento de Antropología y Etnología de América-Facultad de Filosofía y LetrasUniversidad Complutense de Madrid (Cuadernos de Antropología Social y Etnología, núm. 2), Madrid.

— 1972, "El indigenismo como acción social en América”, en Documentación Social. Revista de Desarrollo Social, núm. 7, julio-septiembre, Madrid. , 1973, "Notas para una tipología de comunidades rurales", en Primera Reunión de Antropólogos Españoles, Departamento de Antropología y Etnología de América-Facultad de Filosofía y Letras-Universidad Complutense de Madrid (Cuadernos de Antropología Social y Etnología, núm. 6), Madrid, pp. 79-97.

,2000, "Las nuevas comunidades mexicanas en los espacios rurales de los Estados Unidos de América”, en Virginia García Acosta (coord.), La diversidad intelectual. Ángel Palerm in memoriam, Centro de Investigaciones y Estudios Superiores en Antropología Social, México, pp. 63-112.

, 1994, "La intersección del agua y el trabajo en la modernísima agricultura californiana”, en Juan Romero y Carlos Giménez (eds.), Regadíos y estructuras de poder, Instituto de Cultura "Juan Gil Albert", Diputación de Alicante, Alicante, pp. 121-150.

, 1997, Los nuevos campesinos, Universidad Iberoamericana, México.

Paul, Benjamin, 1953, "Interview Techniques and Field Relationships", en Anthropology Today, Alfred Kroeber, Chicago.

Pérez del Olmo, Fernando, 1987, "Informe de comunidad: Gascones”, Seminario de Investigación Antropológica, Universidad Autónoma de Madrid, Madrid.

_, 1989, "Modos de adaptación tecnoeconómica de las explotaciones ganaderas familiares de la Sierra 
Norte de Madrid", tesis de licenciatura, Departamento de Sociología y Antropología Social, Universidad Autónoma de Madrid, Madrid.

Ramírez, Ángeles, 1987, "Informe de comunidad: Villavieja de Lozoya”, Seminario de Investigación Antropológica, Universidad Autónoma de Madrid, Madrid.

— 1990, "La tierra y las relaciones sociales en un área periurbana: el caso de la Sierra Norte de Madrid”, tesis de licenciatura, Departamento de Sociología y Antropología Social-Universidad Autónoma de Madrid, Madrid.

Rionda, Luis Miguel, 1980, "La tenencia comunal de la tierra en el pueblo de San Nicolás Coatepec, Estado de México", informe de práctica de campo, Proyecto "Movilidad social y ocupacional del campesino en México", Universidad Autónoma Metropolitana-Iztapalapa, México, mecanoescrito.

Rivers, William H. R., 1968, Kinship and Social Organization, Together with the Genealogical Method of Anthropological Inquiry, Athlone Press, Londres [1910, “The Genealogical Method of Anthropological Inquiry”, en The Sociological Review, vol. 3, pp 1-12].

Romero, Juan y Carlos Giménez (eds.), 1994, Regadíos y estructuras de poder, Instituto de Cultura "Juan Gil Albert”, Diputación de Alicante, Alicante.

Roca-Sastre Muncunill, Ramón (ed.), 1977, La región y el desarrollo, Dopesa, Madrid.

San Bruno, Patrocinio, 1987, "Informe de comunidad: Lozoya del Valle”, Seminario de Investigación Antropológica, Universidad Autónoma de Madrid, Madrid.

Steward, Julian, 1950, Area Research: Theory and Practice, Social Science Research Council, Nueva York.

—, 1953, Theory of Culture Change, University of Illinois Press, Vibana.

— 1968 , "Levels of Sociocultural Integration: An Operational Concept", en Robert Manners y David Kaplan (eds.), Theory in Anthropology, Aldine, Chicago. , 1992, “Teoría y práctica del estudio de áreas”, en Jacinta Palerm Viqueira (ed.), Guía y lecturas para una primera práctica de campo, Universidad Autónoma de Querétaro, Querétaro, pp. 11-240.

- et al., 1955, The People of Puerto Rico: A Study in Social Anthropology, University of Illinois Press, Urbana.

Suanzes, Cristina, 1987, "Informe de comunidad: Prádena del Rincón”, Seminario de Investigación Antropológica, Universidad Autónoma de Madrid, Madrid.

Urquiola, José Ignacio, 1994, “Riego, producción y control de mano de obra: formación de una región agrícola en la Nueva España”, en Juan Romero y Carlos Giménez (eds.), Regadíos y estructuras de poder, Instituto de Cultura "Juan Gil Albert", Diputación de Alicante, Alicante, pp. 73-100.

Viqueira, Carmen, 1991, "Prólogo”, en Carlos Giménez, Permanencia y funcionalidad del régimen comunal agrario en España y México, Ministerio de Agricultura, Pesca y Alimentación, Madrid, pp. 13-15.

_ 1994, "La aplicación de la teoría del despotismo oriental a Mesoamérica”, en Juan Romero y Carlos Giménez (eds.), Regadíos y estructuras de poder, Instituto de Cultura "Juan Gil Albert", Diputación de Alicante, Alicante, pp. 53-72.

_ 1999, "Algunas experiencias sobre la enseñanza de la antropología social en México", en Áreas. Revista de Ciencias Sociales, núm. 19.

_ 2000, "Algunas aportaciones de Ángel Palerm a la enseñanza de la antropología”, en Virginia García Acosta (coord.), La diversidad intelectual. Ángel Palerm in memoriam, Centro de Investigaciones y Estudios Superiores en Antropología Social, México, pp. 115-128.

— 2001, El enfoque regional en antropología, Universidad Iberoamericana, México.

Warman, Arturo, 1973a, Los campesinos. Hijos predilectos del régimen, Nuestro Tiempo, México.

—, $1973 \mathrm{~b}$, Ensayos sobre el campesinado mexicano, Nueva Imagen, México.

Wittfogel, Karl, 1966, El despotismo oriental, Guadarrama, Madrid.

Wolf, Eric, 1966, Los campesinos, Labor, Barcelona, 1966 [Peasants, Prentice-Hall (Foundations of Modern Anthropology Series), New Jersey].

_ 1967a, "Types of Latin American Peasants", en George Dalton (ed.), Tribal and Peasant Economies, The Natural History Press, Nueva York.

— 1967b, "Closed Corporate Peasant Communities in Mesoamerica and Central Java”, en Jack M. Potter, May Díaz y George Foster, Peasant Societies: A Reader, Little Brown (The Little Brown Series in Anthropology), Boston.

— 1967c, "Levels of Communal Relations", en Mash Manning (ed.), Handbook Middle American Indians, University of Texas Press, Austin, pp. 299-316.

_ 1972, Las luchas campesinas del siglo XX, Siglo XXI, Madrid.

- 1982, Europe and the People without History, University of California Press, Berkeley, Los Ángeles y Londres. 